\title{
Increased IL-1及 activation, the culprit not only for defective insulin secretion but also for insulin resistance?
}

\author{
Marianne Böni-Schnetzler ${ }^{1}$, Marc Y Donath ${ }^{1}$ \\ IDivision of Endocrinology, Diabetes and Metabolism and Department of Biomedicine, University Hospital Basel, Basel, Switzer- \\ land \\ Cell Research (2011) 21:995-997. doi:10.1038/cr.2011.85; published online 24 May 2011
}

Type 2 diabetes is a chronic progressive disease characterized by insufficient insulin secretion to compensate for insulin resistance. The onset of type 2 diabetes and its progression are mainly determined by the progressive failure of the pancreatic islet $\beta$-cells to produce sufficient levels of insulin. In contrast, insulin resistance is typically present throughout the progression from prediabetes to the later stages of type 2 diabetes. Increasing evidence associates metabolic disorders, including obesity and type 2 diabetes, with inflammatory processes characterized by elevated production of proinflammatory cytokines and infiltration of immune cells [1]. Recently, three studies were published pointing to a role for the IL-1 $\beta$ pathway in insulin resistance [2-4]. In the past, the cytokine TNF $\alpha$ has been associated with insulin resistance in liver and fat tissues [5], while IL-1 $\beta$ was linked to defective insulin secretion in pancreatic islets of type 2 diabetics [6]. In a clinical proof of concept study, attenuation of IL-1 $\beta$ activity with the receptor antagonist IL-1Ra demonstrated the importance of the innate immune system in glycemic control and identified the pancreatic islet as a prime target for IL-1 intervention [7]. Of note, while

Correspondence: Marc Y Donath

E-mail: MDonath@uhbs.ch $\beta$-cell secretory function improved, changes in peripheral insulin sensitivity were not observed. Similarly, in a recent study with prediabetic patients, IL-1 antagonism improved insulin secretion, but again insulin sensitivity remained unaffected [8]. Using rodent models lacking either the inflammasome component Nalp3, or Pycard, or caspase 1 or its substrate proIL- $1 \beta$, the three above-mentioned studies now convincingly show that IL- $1 \beta$ is also linked to the development of insulin resistance [2-4].

Inflammasomes are danger-sensing multiprotein platforms, which promote auto-catalytic activation of the cysteinprotease caspase 1 required for processing of inactive proIL-1 $\beta$ into active IL-1 [9]. Stienstra et al. demonstrated that mice lacking caspase 1 or IL- $1 \beta$ or Nalp3 have improved insulin sensitivity, thereby linking the inflammasomes to insulin resistance [2]. Genetic ablation of inflammasomes not only resulted in increased insulin sensitivity but also regulated adipocyte development and protected from the negative effects of diet-induced obesity. Interestingly, similar to islet $\beta$-cells [6], adipocytes themselves produce IL-1 $\beta$. This initial event may then lead to IL-1 $\beta$-induced chemokines, resulting in the recruitment of macrophages [10-12]. Subsequently, macrophage influx and activation within fat tissue may act as a prominent source of IL- $1 \beta$ secretion, triggering insulin resistance. Along this line the papers by B. Vandanmagsar et al. [3] and by H. Wen et al. [4] take this observation a step further and examine possible mechanisms of inflammasome activation and release of IL- $1 \beta$ in macrophages during obesity. Both confirm the role of Nalp3 in insulin resistance, but in contrast to the observation by Stienstra et al. [2], the protective phenotype was only evident in Nalp3 knockout mice fed a high-fat diet while mice on chow diet did not have improved insulin sensitivity. To gain insight into the mechanism of inflammasome activation in macrophages, both groups used bone marrow-derived macrophages primed in vitro with Lipopolysaccaride (LPS). LPS activates Toll like receptor 4 (TLR4) and this priming leads to increased transcription of IL-1 $\beta$ mRNA. As a second trigger for the LPS-primed macrophages Vandanmagsar et al. used ceramides [3], the specific product of long-chain saturated fatty acid metabolism while Wen et al. directly used the saturated free fatty acid (FFA) palmitate [4]. Both led to elevated caspase 1 activation and IL- $1 \beta$ release in macrophages from wild-type mice but not in macrophages from Nalp3 or Pycard knockout mice. Interestingly, Wen et al. further show that LPS-palmitate stimulation uses an AMP-activated protein kinase (AMPK)-reactive oxygen species (ROS) pathway to activate the 
Nalp3 inflammasome, whereby ROS is derived from mitochondria. Of note, the widely used anti-diabetic drug metformin attenuates AMPK and thus may act via reduction of IL-1 $\beta$ activity. FFA-mediated IL- $1 \beta$ formation has also been observed in pancreatic mouse and human islets [13], but unlike in macrophages, FFAs alone are capable to induce IL- $1 \beta$ release in islets and a further stimulation is observed in the presence of enhanced glucose levels. As elevated glucose concentration defines diabetes, it would be interesting to test whether glucose also promotes IL- $1 \beta$ release in macrophages or whether this is a path- way specific for islets that are capable of sensing glucose. Alternatively, high-fat diet was reported to lead to elevated circulating LPS levels [14] and this may prime the inflammasomes in macrophages and render them responsive to elevated FFA levels in prediabetic obese individuals (Figure 1).

As both master cytokines IL- $1 \beta$ and TNF $\alpha$ are implicated in insulin resistance [2-5], Wen et al. used either the receptor antagonist IL-1 Ra to block IL-1 activity or an anti-TNF antibody to inhibit TNF and show that they partially reversed the inhibition of insulin signaling mediated by macrophage-condi- tioned media. Full restoration of insulin signaling was only obtained when IL1 Ra was used in combination with the anti-TNF antibody. Interestingly, the role of IL-1 $\beta$ in macrophage-mediated insulin resistance is further supported by a reduced ability of Pycard-deficient bone marrow cells to deteriorate glucose tolerance in bone marrow chimera experiments (Wen et al., Figure 8f). This suggests that macrophage influx could indeed mediate insulin resistance via activation of the inflammasomes. A limit to this conclusion is the lack of insulin secretion data to rule out the possibility that the impairment of glucose tolerance

\section{Insulin action adipocytes}

\section{Insulin production islet $\beta$-cell}

\section{tissue macrophages}

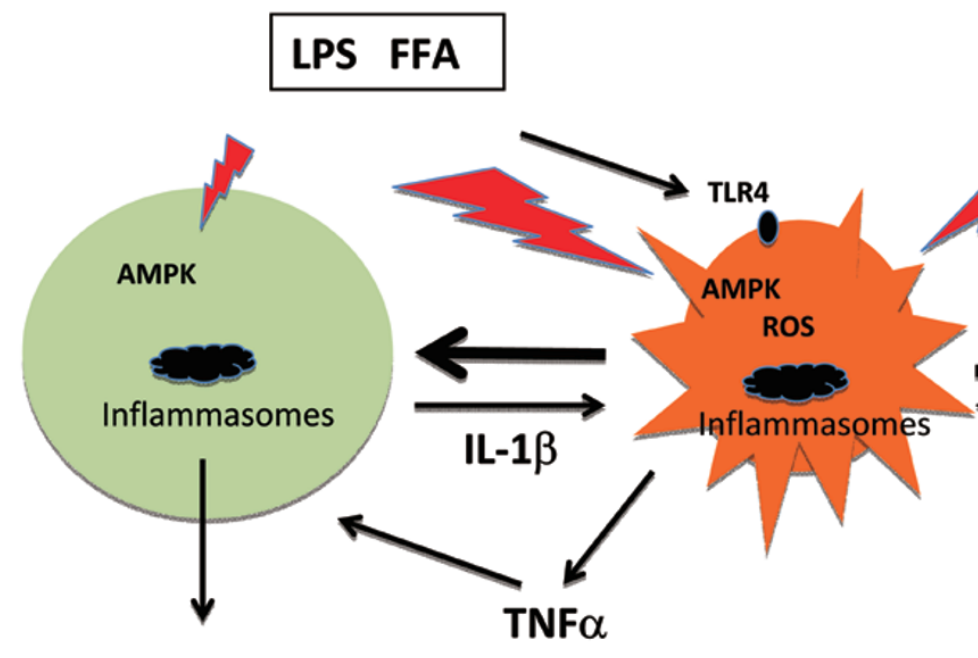

Chronic Inflammation

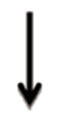

Insulin resistance $\beta$-cell dysfunction and death

Figure 1 Inflammasome-mediated IL-1 $\beta$ production impairs glucose homeostasis by inducing insulin resistance and $\beta$-cell dysfunction. Metabolic stress activates inflammasomes in islets and in insulin-responsive tissues, leading to the presence of activated macrophages and resulting in an inflammatory state. Two "hits" by LPS and FFA are required in macrophages to activate the inflammasomes via AMPK and subsequent mitochondrial ROS generation. In $\beta$-cells FFA and elevated glucose concentrations lead to IL-1 $\beta$ release, while inflammasomes are activated via glucose-induced dissociation of thioredoxin-interacting protein (TXNIP) from thioredoxin under the influence of ROS. The chronic inflammation in insulin-responsive tissues leads to elevated TNF $\alpha$ and IL-1 $\beta$ that mediates insulin resistance while IL-1 $\beta$ in islets impairs the function of the pancreatic $\beta$-cell. 
by transferred macrophages is due to effects on islet function.

Regardless of the precise underlying mechanism, an impressive number of genetic animal models now demonstrate a role for the inflammasome in mediating IL-1 $\beta$-induced insulin resistance. This is further supported by earlier intervention studies showing that IL-1 blockade in animal models of diabetes improved both insulin secretion and sensitivity [15]. This should help to design further clinical studies aiming at improving not only insulin secretion but also insulin action via modulation of the IL- $1 \beta$ system. Thereby, the sensitivity of the islets to IL- $1 \beta$, which express the highest level of IL-1 receptors [13], and possible differences in the pharmacokinetics due to the strong blood flow of the islets have to be taken into account. Thereby, novel long-acting IL- $1 \beta$ antagonists may be more efficient than IL-1Ra.

\section{References}

1 Donath MY, Shoelson SE. Type 2 diabetes as an inflammatory disease. Nat Rev Immunol 2011; 11:98-107.

2 Stienstra R, Joosten LA, Koenen T, et al. The inflammasome-mediated caspase- 1 activation controls adipocyte differentiation and insulin sensitivity. Cell Metab 2010; 12:593-605.

3 Vandanmagsar B, Youm YH, Ravussin A, et al. The NLRP3 inflammasome instigates obesity-inducedinflammation and insulin resistance. Nat Med 2011; 17:179-188.

4 Wen H, Gris D, Lei Y, et al. Fatty acidinduced NLRP3-ASC inflammasome activation interferes with insulin signaling. Nat Immunol 2011; 13:408-415.

5 Wellen KE, Hotamisligil GS. Inflammation, stress, and diabetes. J Clin Invest 2005; 115:1111-1119.

6 Maedler K, Sergeev P, Ris F, et al. Glucose-induced beta-cell production of interleukin-1beta contributes to glucotoxicity in human pancreatic islets. J Clin Invest 2002; 110:851860.

7 Larsen CM, Faulenbach M, Vaag A, et al. Interleukin-1-receptor antagonist in type 2 diabetes mellitus. $N$ Engl J Med 2007; 356:1517-1526.

8 van Asseldonk EJ SR, Koenen TB, Joosten LA, Netea MG, Tack CJ. Treatment with anakinra improves disposition index but not insulin sensitivity in nondiabetic subjects with the metabolic syndrome: a randomized, double-blind, placebo-controlled study. $J$ Clin Endocrinol Metab 2011 Apr 20; doi:10.1210/jc.2010-2992
9 Martinon F, Burns K, Tschopp J. The inflammasome: a molecular platform triggering activation of inflammatory caspases and processing of proIL-beta. Mol cell 2002; 10:417-426.

$10 \mathrm{Xu} \mathrm{H}$, Barnes GT, Yang Q, et al. Chronic inflammation in fat plays a crucial role in the development of obesity-related insulin resistance. J Clin Invest 2003; 112:1821-1830.

11 Weisberg SP, McCann D, Desai M, Rosenbaum M, Leibel RL, Ferrante AW Jr. Obesity is associated with macrophage accumulation in adipose tissue. J Clin Invest 2003; 112:17961808.

12 Ehses JA, Perren A, Eppler E, et al. Increased number of islet-associated macrophages in type 2 diabetes. Diabetes 2007; 56:2356-2370.

13 Boni-Schnetzler M, Boller S, Debray $\mathrm{S}$, et al. Free fatty acids induce a proinflammatory response in islets via the abundantly expressed interleukin-1 receptor I. Endocrinology 2009; 150:5218-5229.

14 Cani PD, Amar J, Iglesias MA, et al. Metabolic endotoxemia initiates obesity and insulin resistance. Diabetes 2007; 56:1761-1772.

15 Ehses JA, Lacraz G, Giroix MH, et al. IL-1 antagonismreduces hyperglycemia and tissue inflammation in the type 2 diabetic GK rat. Proc Natl Acad Sci USA 2009; 106:13998-14003. 